\title{
Approaches of Buried Object Detection Technology
}

\author{
Nagashree $\mathrm{R} \mathrm{N}^{\mathrm{a}^{*}}$, Aswini $\mathrm{N}^{\mathrm{b}}$ \\ ${ }^{a} P G$ student in Digital Electronics \& Communication, MVJCE, Bengaluru and 560067,India. \\ ${ }^{b}$ Assiscant Professor in Dept of Electronics \& Communication, MVJCE, Bengaluru and 560067,India.
}

\begin{abstract}
This paper describes the different art of buried object detection technology and algorithms. This detection of buried object finds application in many areas, importantly in the Landmine detection which is of growing concern due to the danger of buried landmines to people's lives, economic growth and development. This paper describes and analyzes different technology available. The approaches discussed are Electrical Impedance Tomography, X-ray backscatter, Infrared Systems, Acoustics/seismic systems; Neutron based Method and finally Ground-Penetrating Radar with two commonly available approaches: Least squares and SVD approach. Finally, the paper concludes highlighting the need to improve the way this information is processed and compared.
\end{abstract}

Index Terms: Electrical Impedance Tomography, X-ray backscatter, Infrared Systems, Acoustics/ seismic systems, Neutron Methods, SVD, Least squares.

(C) 2014 Published by MECS Publisher. Selection and/or peer review under responsibility of the Research Association of Modern Education and Computer Science

\section{Introduction}

Today, the subsurface buried object detection is of growing concern as it finds a wide and important application in military field for detection of mines. And as now a day the metal content is being very low in landmines, making metal detector inappropriate choice in detection and thus has become a challenging task. This paper describes difficulties of predicting the potential for mine detection system to reduce the false alarm rate and increase the probability of detection is an inherently difficult task.

A number of innovative methods are being explored that search for buried mines based on changes in the Electromagnetic properties of the surface soil and shallow subsurface. These methods include ground Penetrating Radar (GPR), Electrical Impedance Tomography (EIT), X-ray Backscatter, and Infrared systems. Some of the other methods discussed are Acoustic/seismic system and Neutron based system.

\section{Technologies for Buried Object Detection}

* Nagashree R N. Mob.: 7259544509

E-mail address: nagashree.r.n@gmail.com 
The concept concentrated in this paper is based on the object is buried under ground. The Fig 1. Shows the block diagram of ground buried target along with incident, reflected and scattered wave. The transmit antenna transmits the signal which strikes the air-ground interface and part of signal is reflected back to air medium whereas part of signal travels into the ground medium and strikes the target and some signals reflects back and some of them are scattered back which are considered as signal of interest that are processed to get the detection and location information.

The discussed approaches consider different properties of the ground, target and environment etc. to detect and locate the object.

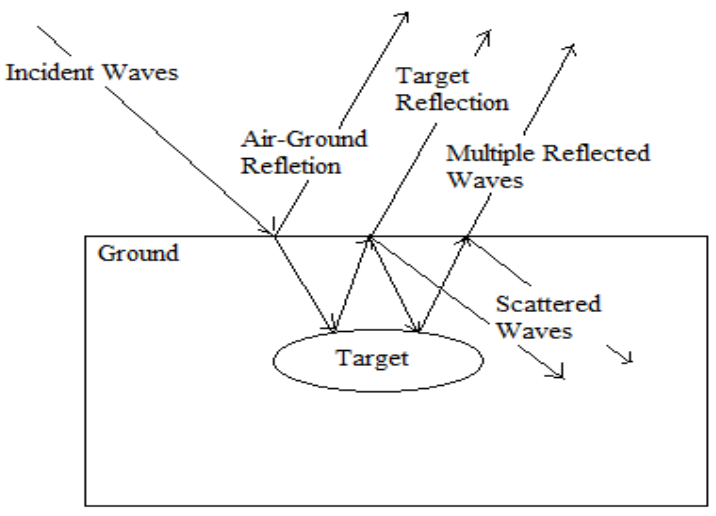

Fig 1. Signal reflection and scattering.

\subsection{Electrical Impedance Tomography}

Electrical Impedance Tomography approach uses electrical currents to image the conductivity distribution of the medium under investigation. EIT is used in geophysics for the detection of mines. This approach uses a two-dimensional array of electrodes are placed on the ground surface to collect the signals which is the conductivity information of the stimulations of pair wise combinations of electrodes, which in turn gives the information about presence of objects such as mines. The data obtained is then post processed with an algorithm that renders an image of the conductivity profile of the subsurface volume.

This system allows detection both of metallic and non-metallic objects due to conductivity anomalies that produce images. Thus providing the information about the detection and location of object.

Some of the advantages of this approach are it behaves well in wet soil and as the objects buried can be both metallic and non-metallic. It also behaves well in wet soil and the equipment is relatively simple, cheap and light.

A disadvantage is that sensors must be in contact with the surface, therefore increasing the risk of detonation. They do not work well in dry and nonconductive environment like desert or rocky surfaces. Moreover, the performance falls down substantially with the depth of the object being detected for fixed electrode array size, generally making it appropriate only for shallowly buried objects. The technology is also sensitive to electrical noise. This technology is used mostly in medical field and industrial process imaging.

\section{$2.2 \quad X$-ray backscatter}

The familiar application of $\mathrm{x}$-ray is in medical field to produce an image of an object. The same concept is also applied to the detection of buried object.

X-ray backscatter (XBT) has the potential for low false alarm rates and high detection probability. X-ray 
radiography produces an image of an object by passing photons through the object. The X-rays have a very small wavelength compared to the ground buried objects such as mines, so the photons can easily pass through the object to produce high - quality images of mines. Although it is difficult for the x-rays to physically penetrate and to employ the imaging, the backscatter of $x$-rays will provide information about buried, irradiated objects.

The characterization of the return spectrum through energy or spatial density or temporal distribution can be used to distinguish material distribution in buried object, i.e., this approach employed based on the fact that different materials possess different mass densities, as for the case of buried landmines, the soil and mines and possess slightly different mass densities and effective atomic numbers that differ by a factor of about two.

There are two basic approaches that can be employed to use the backscattered $\mathrm{x}$ rays to create images of buried mines. First, the methods that collimate (i.e., align) the $\mathrm{x}$ rays to employ the focused beams and collimated detectors to form an image. But this collimation process increases size and weight and dramatically reduces the number of photons available for imaging. Thus, high-power x-ray generators are used as sources. The large size, weight, and power requirements of such systems are not suitable to person-portable detectors. Second, uncollimated methods illuminate a broad area with $\mathrm{x}$ rays and then use a spatial filter to deconvolve the system response. They may be suitable for person-portable detection.

The use of XBT technology has advantages such as the scatter signal is directly proportional to the density of the material in the irradiated volume, it requires only single-sided access, and high image contrasts are achievable, therefore that XBT technology has a high potential for imaging purposes.

The use of this technology is limited by the depth of the mines, and hence detection is limited only to shallow buried objects. And in the required energy range, penetration of x-ray scatter device into soil is poor. If source strengths are kept low enough to be safe for a person-portable system, the time required to obtain an image may be impractically long.

The $\mathrm{x}$-ray backscatter albedo techniques are ineffective of detection of mines and IED because of high false alarm rates due to surface irregularities and detector height variations. To overcome this, the technique called coded aperture x-ray backscatter imager for IED detections are employed.

\subsection{Infrared Systems}

In Infrared methods detection approach is based on the variations in electromagnetic radiation reflected or emitted by either surface mines or the soil and vegetation immediately above buried objects.

It is based on diverse characteristic such as active and passive irradiation using a broad range of electromagnetic wavelengths. The two categories of infrared systems are thermal and non-thermal, Thermal detection methods exploit diurnal variations in temperatures of areas near mines relative to surrounding areas. That is the buried objects such as mines and the soil above them tend to be warmer than surrounding areas where no object is buried during the day and gets cooled more quickly at night. For non-thermal detection methods depends on the fact that areas near mines reflects the light differently than surrounding areas.

Anthropogenic materials tend to preserve polarization because of their characteristically smooth surfaces, allowing discernment of surface mines. Moreover, the physical activity of emplacing mines changes the natural soil particle distribution by bringing small particles to the surface, which in turn affects the way in which the soil scatters light. Systematic changes in vegetation moisture levels immediately above buried mines also may be leveraged.

The infrared systems are found to be more attractive than other approaches as the surface irregularities and effects of physical contact do not affect the system. Thus eliminating the clutter related to non-uniform surface. And the system is found to be of lighter weight, and is more effective to scan wide range on area relatively quick. Hence the image acquisition is fast.

This approach is limited as its performance is variable and depends on characteristics of the environment and hence need a close detection and accurate identification of object location also the technologies are able to detect buried mines under only limited transient conditions. Even though the system uses good and high 
accurate sensor, the algorithms used to process the signals are comparatively undeveloped are not linked to physical phenomena hence most of the authors says that the sensors used in this approach need to improve. Thermal signatures are not well understood, and a comprehensive predictive model does not exist.

\subsection{Acoustics/ seismic systems}

Acoustics and seismic systems emit the sound waves into the ground in order to get back the vibration from it. It is based on the fact that the different materials with different properties vibrate differently when exposed to sound waves. When the emitted sound waves strikes the ground surface some of the acoustic energy reflects off the surface, but the rest of the waves penetrates the ground and propagate through the soil. And when these waves strike the buried object such as mines again some of the energy reflects upward toward the ground surface, causing vibration at the surface.

The specialized sensors detect and capture the reflected waves from the soil and the mines, and analyze for the difference in amplitude and frequency that makes detection possible and thus complement the information obtained from EMI sensors. The sensors detect the vibration without the need of contacting the surface. Some of the different kinds of sensors are laser Doppler vibrometers, radars, ultrasonic devices, microphones.

These acoustic/seismic sensors are based on physical effects and thus are different from the other sensors. For example, they sense differences in mechanical properties of the mine and soil, while GPR and EMI sensors detect differences in electromagnetic properties. The main strength of this system is its potential of having very low false alarm rates. As false alarms from naturally occurring clutter, such as rocks and scrap metal, have been extremely low. An additional advantages is that, unlike GPR systems, these sensors are unaffected by moisture and weather, although frozen ground may limit the sensor's capability.

The acoustic/seismic systems are unable to detect the mines that are deeply buried in the ground thus limiting the detection range to shallow buried object as resonant response attenuates significantly with depth. The slow speed of existing systems is also one of the limitations. Speed currently is limited by the displacement sensor, which senses the vibrations at the surface caused by the sound waves. These displacements are very small (less than $1 \mu \mathrm{m}$ ) and are thus difficult to measure quickly in the adverse conditions of a minefield. The required scan time for locating antipersonnel mines may range from 125 to 1,000 seconds per square meter.

\subsection{Neutron Methods}

The Neutron based method involves the technique of distinguishing the buried object such as mines from the surrounding soil materials by probing the soil with neutrons and detecting the reflected neutrons. The difference in characteristics such as intensity and energy of the reflected radiation indicates the presence of buried objects.

The advantage of this approach is the physical properties of neutron moderation allow the technology to use low-strength source radiation, which reduces shielding required to protect workers from radiation exposure. Thus, designing a handheld system may be possible. Costs of a production imager are expected to be moderate. The neutron backscatter imagers, exploiting the neutron signal eliminates the problems associated with nonimaging detectors.

The Limitations of Neutron based approach is that the activation methods can, at best, measure relative numbers of specific atoms but cannot determine what molecular structure is present. Because neutron moderation is sensitive to water, may produce false alarms. And they also produce high false alarm rates due to soil moisture variations, surface irregularities and detector height variations. Ground-surface fluctuations and sensor height variation also contribute to false alarms in non-imaging systems. Imaging can reduce these effects, although some degradation of the image is expected.

\subsection{Ground Penetrating Radar}


Ground Penetrating Radar uses the radar signal to detect the subsurface structures such as rock, ice pavement and structure etc., by emitting radio waves into ground. The GPR can detect the variations in dielectrics and when the transmitted waves strikes the object or boundary of different dielectric constants and the waves gets reflected and these reflected signals are analyzed.

In our application GPR system is mainly used for detecting the buried objects and it consist of an antenna or series of antennas that transmit the waves and then pick up the return signal. The signal processing system is a small computerized system interprets the return signal to determine the object's shape and position. The quality of the image will be better if size of the object is proportional to the wavelength of the input signal i.e. as the wavelength decreases and the frequency increases, can get a better resolution image. But at the higher frequencies the penetration of the signal into the ground surface will be poor. Therefore the designer must compromise between the quality of the image and penetration depth. The system has three types of displays of surface data, including: a one-dimensional trace (A-scan), two-dimensional cross section (B-scan) and threedimensional display (C-scan).

Today the GPR is widely being applied in the military applications for the detection of mines and under the favorable conditions the can even provide information concerning the nature of the buried objects. And many research works are going on to provide a 3-D pseudo image of the subsurface. However the detection of buried object becomes very complex when clutter is present. The clutter varies according to the irregularities of the ground surface and the soil conditions therefore performance of GPR depends on the knowledge of the prevailing weather conditions, soil type and soil water content.GPR performance can be highly sensitive to complex interactions among mine metal content, interrogation frequency, soil moisture profiles, and the smoothness of the ground surface boundary.

There are basically two GPR systems: vehicle mounted and handheld. The Vehicle mounted systems produce more reliable signatures by keeping both the detector speed and height constant. In handheld systems, a human operator sweeps the detector over the ground surface. In this case, constant detector speed and height assumption is not valid. Hence, inconsistent object signatures can be observed, which need to be balanced with appropriate signal processing techniques.

The GPR has a number of advantages such as, it is complementary technique to the conventional metal detectors, as it senses changes in the dielectric constant and then finds mines with a wide variety of types of casing containing plastic or other material (not those with metal). Generating an image of the mine or other buried object based on dielectric constant variations is often possible because the required radar wavelength is generally smaller than most mines at frequencies that still have reasonable penetration depth. Second, GPR can be made light weight and easy to operate, and it scans at a rate comparable to that of an EMI system.

The Limitations of GPR are natural subsurface inhomogeneities (such as roots, rocks, and water pockets) can cause the GPR to register return signals that resemble those of landmines and thus are a source of false alarms. In addition, GPR performance can be highly sensitive to complex interactions among mine metal content, interrogation frequency, soil moisture profiles, and the smoothness of the ground surface boundary. GPR will perform poorly in wet soils for landmines buried below a depth of about $4 \mathrm{~cm}$. However, theoretical investigations indicate that increased soil moisture and interrogation frequency may actually strengthen the return signal for nonmetallic mines, but non-uniform soil moisture profiles and rough ground surfaces present difficulties. For the same mine, a given GPR can be very effective or ineffective, depending on soil moisture and mine location; such complex interplays make performance highly variable and difficult to predict. An additional limitation is that unless the GPR system is tuned to a sufficiently high frequency, it will miss very small plastic mines buried at shallow depths because the signal "bounce" at the ground surface (caused by the electrical property differences between air and soil) will mask the return signal from the mine. Finally, the GPR system designer must make a tradeoff between resolution of the return signal and depth, because highfrequency signals yield the best resolution but do not penetrate to depth.

The two commonly used algorithms employed in GPR are discussed here:

1) Least Square Approach to Buried Object Detection using Ground Penetrating Radar Handheld Systems 
Detecting a buried object using GPR involves scanning the same ground surface and evaluation of differences between consecutive GPR signals taken at adjacent points. Each 1D GPR signal taken at a specific point is called an A-scan. Hence, a detection algorithm should distinguish between A-scans taken in the presence and absence of a buried object. From the A-scans taken in the absence of a buried object, the next Ascan should be estimated. After measuring the GPR signal at that point, the observed signal and the estimated signal should be similar. Otherwise, the presence of a buried object can be concluded.

In least squares approach, using some of the previous A-scans, the next A-scan is to be estimated. Previous A-scans must correspond to clutter samples for the algorithm to work correctly, as the detection is based on the difference between clutter responses and buried object responses. At each different site, the starting point of the inspection is assumed to be target-free and a few A-scans are recorded to be used in the estimation. If this assumption is not valid, the detector will give alarm for all clutter samples as the operator sweeps the handheld detector, which will warn the operator to record the clutter samples to be used in the estimation again.

Least squares formulation results in a system of linear equations at each point. To achieve real-time performance, Cholesky factorization is used when solving these linear equations. Experiments show that the proposed algorithm provides almost $100 \%$ performances at a FAR of $10 \%$, when ten A-scans are used to estimate the next A-scan. Thus the Least Squares approach high probability of detection of the buried target without the high FAR.

\section{2) Optimized Singular Value Decomposition Approach for the Detection of Targets from Ground-Penetrating Radar Data}

In the optimized SVD approach B-scan is employed to record the two dimensional value over the surface. Here two-pass ground-penetrating radar (GPR) data are recoded pass-1(written as s1) corresponds to scanning the ground without target buried and pass-2(written as s2) scanning corresponds to that of with target buried. The pass- 1 data thus considered as reference signal and it is subtracted from pass- 2 data, if it results in a considerable amount difference, implying the presence of target.

The temporal dependences of the measured data are explicitly written as $\mathrm{s}(\mathrm{xl}, \mathrm{i}, \mathrm{yl}, \mathrm{j}, \mathrm{tl}, \mathrm{k})$, where $\mathrm{l}=1,2$ is the pass number, $\mathrm{xl}, \mathrm{i}$ and $\mathrm{yl}, \mathrm{j}$ are the along- and cross-track coordinates, respectively, of the receivers for each pass ( $\mathrm{i}=1, \ldots, \mathrm{N}$ and $\mathrm{j}=1, \ldots, 21$, where $\mathrm{N}$ is the number of data points in the along-track direction), and tl,k is the time sequence for each pass recorded by the GPR over 800 samples $(\mathrm{k}=1, \ldots, 800)$. Ideally, $\mathrm{xl}$, yl, and $\mathrm{tl}$ are independent of 1 , but realistically, the sets of points over which measurements are collected are different.

The matrices for SVD are constructed based on channel diversity across time bins and are denoted as sl[i], l= $1,2, \mathrm{i}=1 \ldots \mathrm{N}$, with size $[800 \times 21]$. The associated SVD matrices are written as

$$
\begin{aligned}
& \mathrm{s}_{1}=\mathrm{U}_{1} \Sigma_{1} \mathrm{~V}_{1} \\
& \mathrm{~s}_{2}=\mathrm{U}_{2} \Sigma_{2} \mathrm{~V}_{2}
\end{aligned}
$$

The presence of a target, i.e., a coherent scatterer, should be mostly revealed in the matrix $\Sigma$ as new Eigen values and not in the basis matrices $\mathrm{U}$ and $\mathrm{V}$.For two passes over the same ground, we expect similar bases, i.e., $\mathrm{U} 1=\mathrm{U} 2$ and $\mathrm{V} 1=\mathrm{V} 2$. The fact that this is not the case with our measured data is mostly due to pass differences. The method is further optimized from a purely mathematical point of view by enhancing the target features so as to create a maximum cancellation dip when compared to the reference signal (obtained without a target).

Here the least square approach is described for A-scan data and Optimized SVD approach for B-scan data. In order to overcome some of the challenges and to remain close to realistic terrain conditions, the SVD approach is limited to the detection of newly implanted subsurface dielectric scatterers, with the underlying assumption that the terrain of interest can be characterized before being contaminated. 


\section{Conclusion}

Humanitarian demining continues to be a world problem far from being solved. We find that there is no standalone system which can detect landmine efficiently. There are many approaches found but their direct results cannot be generalized. But every method has good results within its own limited conditions. And hence there is work to be done in fusion of landmine detection technology in order to enhance its performance. Due to the limitations that are mentioned with each system, there is a need for the development of a multi-sensor system based on type of signal and algorithm, rather than focusing on single efficient technology working independently. Mine detection research and development should emphasize the design from basic principles and further subsequent development and then integrating multisensory system that would overcome the limitations of any single-sensor technology. Thus combining different kinds of sensors would certainly result in an improved and better system in landmine detection. All this compels us to conclude that a single system should be produced.

\section{References}

[1] I. Robledo, M.Carrasco and D. Mery, "A survey of land mine detection technology" International Journal of Remote Sensing Vol. 30, No. 9, 10 May 2009, 2399-2410.

[2] Ahmet Burak, Yoldemir and Mehmet Sezgin, "A Least Squares Approach to Buried Object Detection Using Ground Penetrating Radar”, IEEE Sensors Journal, vol. 11, no. 6, June 2011.

[3] Tomasz M. Grzegorczyk, Beijia Zhang, and Matthew T. Cornick, "Optimized SVD Approach for the Detection of Weak Subsurface Targets From Ground-Penetrating Radar Data”, IEEE trans on Geosci. and Remote Sens, vol. 51, no. 3, march 2013.

[4] Jeffrey J. Daniels, "Ground Penetrating Radar Fundamentals", Department of Geological Sciences, the Ohio State University.

[5] Laura E. Gilcrist, Gregory S. Baker, Saravanan Swaminathan, Donald P. Visco, Jr., Ramesh Bharadwaj, Supratik Mukhopadhyay, Krishna Shenai, and Surajit Sen, "Acoustic Interrogation of Soil and Possible Remote Detection of Shallow Buried Inclusions".

\section{Authors Profile}

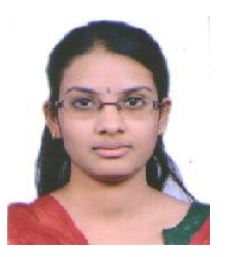

Nagashree R N, Final Year Mtech in Digital Electronics and Enginnering at MVJ College of Engineering. Bachelor of degree was awarded in the field of Telecommunication Engineering in the year 2011 at GSSS Institute of Engineering and Technology for Women. Have teaching experience of 1 year.

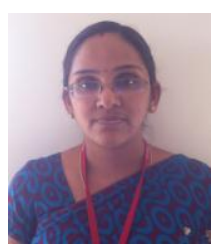

Aswini N, presently works as Assistant professor in Dept. of E\&C at MVJ College of Engineering, Bangalore. She pursued her Bachelors in Applied Electronics \& Instrumentation from College of Engineering, Thiruvananthapuram in 2002 and Masters in VLSI Design and Embedded systems from VTU in 2011. She has 6 years of teaching experience and her areas of interest are in Multi-core processor architectures and VLSI Design. 